\title{
Decision Support Systems Aiming in Reducing Globalization Burdens in Education
}

\author{
Rabih Haddad ${ }^{1,2}$, Eunika Mercier-Laurent ${ }^{1}$ \\ ${ }^{1}$ Computer Science Department, University of Reims Champagne-Ardenne, France, \\ ${ }^{2}$ EPITA Graduate School of Engineering \& Computer Science, Paris, France.
}

\begin{abstract}
The potential drawbacks that globalization might have on education are increasing and becoming a main worry. The number of international students is increasing and students' mobility is becoming a crucial phenomenon to obtain a good degree and secure a decent job. Universities have tripled their efforts to recruit and attract international students but their educational ecosystem is still missing some fundamentals. Ensuring that all international students are admitted using an adapted automated admission system, receiving assistance and decent welcome from international faculty and staff, and building their career after are factors that reduce the negative impacts of globalization in education. This paper presents our Decision Support System using knowledge management (KM) approach and AI techniques contributing to achieve those fundamentals and reduce its impacts.
\end{abstract}

Keywords: Higher Education; Automated Admission System; International Students; Knowledge Management; Text Mining, and Speech Recognition. 


\section{Introduction}

Globalization consisting in the integration of markets, cultures, industries and economies around the world (FT, 2019) affects numerous sectors of human life. One of them is education and more precisely higher education. Regardless of the controversial aspects of its influence on countries, developed or developing, positive or negative it can be an important asset in higher education if it is used in an effective method. In fact, nations, regions, country and cities should manage their intellectual capital (Ordonez, Edvinsson (2015).

Globalization phenomena has been risen in the last 20 years due to increasing of internet penetration rate in the world, the easiness and availability of electronic communications (social media, mobile applications, electronic newsletters...) the growth of the low-cost transportation means and the trend for mobility, included into the EU strategy.

Since globalization involves an integration of various cultures then naturally there should be procedures that govern the impact of globalization in higher education to avoid or at least minimize its drawbacks. This paper presents an analysis on how decision support system using knowledge base assessment can help reducing the following weaknesses:

- Selection and admission of international students.

- Services provided for international students to overcome the cultural differences while pursuing their academic programs.

- Success of international programs, not only among international students but also for the international faculty and staff and the absorption of the potential graduates in the desired markets.

This paper discusses these important drawbacks. Also, it contains our analysis of admission process, brief state of the art, presentation of research method, proposed architecture of DSS (Decision Support System) positioned in the context of educational ecosystems.

\section{Process of Admission of International Students}

With the number of international students increasing globally and the mobility of students is becoming a condition to secure a good job and to gain a shining career, evaluating candidates' prerequisites is becoming challenging (Nguyen, Haddawi, 2007; Bahtiari, 2011). We address this challenge using a methodology based on knowledge management approach powered by adequate AI techniques to help academic institutions in the evaluation of international students' profiles. This methodology should provide multicriteria evaluation of candidates by considering also their cultural background. 
Today applying to any degree seeking program anywhere in the world nationally or abroad requires that candidates should undergo an admission procedure to assess the profile of candidates and to take the final decision (admission/refusal of candidates). Admission systems vary from one country to another and from one institution to another. The whole process is mainly done manually by specialized persons (Min Edu, 2019; Szymankiewicz, 2005) and we did not find any information about a fully automated admission system. The characteristics of each country and institution shape the admission system; most of them require the traditional documentation, evaluation, information: admission and languages proficiency exams, interviews, CVs, transcripts, motivation and recommendations letters. In some countries the first factor of admission is the ability to pay for courses.

An adequate evaluation of students will shorten the gap between the student, the desired country and its educational system. Academic program in any country is designed based on specific cultural aspects and the universal contents of curriculum. Creating an adapted assessment of profiles can predict the eligibility of students to attend an academic program in a multicultural environment.

\subsection{Admission Process}

All recruiting institutions require the candidate information and exams results to decide its admission status. The general admission procedure can be described as follows:

- Students apply online on the institution website by supplying all requested and relevant documents.

- Admission teams process the files following the order:

o Relevance of the candidate to the requested major

o Candidates high school or bachelor grades

o Candidates experience and skills

o Interview conduction (remote or face to face) to detect: genuineness, motivation, and capacity

o Exams conduction to detect knowledge and practice

o Financial status (mainly Anglo-Saxon institutions)

\subsection{Components of Proposed System}

As an entry point to understanding the admission system, we consider two major knowledge blocks contributing to the relevant evaluation of students' profiles: Curriculum Vitae (CV) and the online interview. The CV still is an important document that helps the admission committee identifying important information about the candidates and provides knowledge on student's academic and career path. The online interviews help detecting motivation and validate the consistency and genuineness of the candidates' in line with 
their CVs and profiles. For this purpose, the online interviews will be recorded and rerun for the offline evaluation.

\section{Curriculum Vitae (CV)}

This block is very essential in the evaluation process. In the current systems the CV students are not taken into consideration as the evaluation logic only depends on the profiles of previous students who have succeeded a certain curriculum. Hence it depends on a comparative mechanism that might be valid for students coming from the same background but might fail for a diversified group of students. CV should provide the following information about each candidate:

- Basic: to detect the country of origin of each candidate, age and gender. The country will be a crucial factor in the adapted evaluation since a cultural impact matter here.

- Academic background: to detect the institution attended by the candidate and the highest degree obtained and the number of academic years after high school. This will bring elements for a base of academic institutions worldwide that will be ranked based on students' success after enrollment and pursuing of classes.

- Professional experience: to verify the experience and skills acquired and its relevancy to the degree obtained. This will bring elements for a base of companies worldwide that will be ranked based on students' success after enrollment and pursuing of classes.

Beside the direct knowledge that will be extracted from CVs, text mining will be applied to discover knowledge from the unstructured text by merging all the above listed information.

\section{Online Interview}

The online interview will be used to evaluate the English language level of candidates, their motivation, their capability to present themselves and present a coherent project of life, and some easy behavioral aspects. The video interviews will serve to:

- Detect the candidates' oral ability by evaluating the first couple of minutes of their interview.

- Analyze the candidates' behavior in terms of self-confidence and consistency.

- Evaluate the candidates' answers to detect their motivation and relevance.

The integrated system of student's evaluation using knowledge management approach is presented in Figure 1. The main aim is to assess how KM can help in such system. By presenting and analyzing the 2 major components of the evaluation system, we will be able to obtain an architecture that leads to an adequate evaluation of candidates. Also, this analysis will help us to validate the proposed solution on real cases and integrate feedback. 
Such evaluation is beyond the traditional one assessing the experience, the skills acquired and the behavior in a multinational environment.

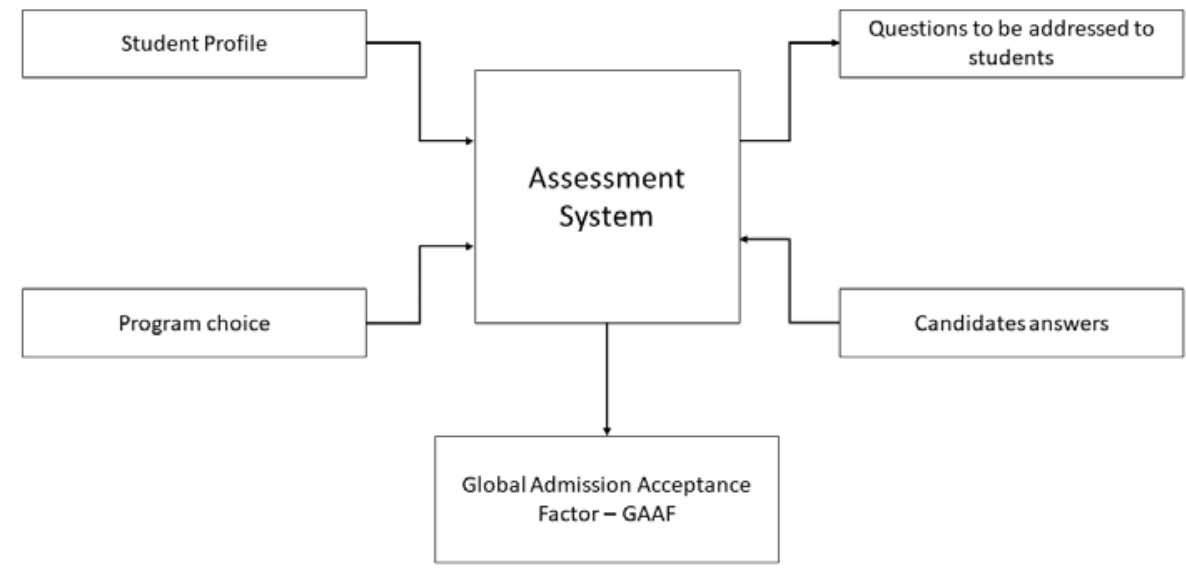

Figure 1. Proposed architecture of decision support system for student admission

This admission system should provide universities with qualified profiles who are adapted to the desired programs and countries and hence reduce the risk of having students integrating international institutions abroad but never managing to validate the academic conditions to get their degree and missing the main objective of coming to these countries.

\section{Innovating Educational Ecosystem - added values and benefits}

As per the UNDP (United Nation Development Program) by following the 4 learning principles (to live together, to know, to do, and to be) many globalization problems such as meeting current social challenges and acquiring new skills in a fast-moving technological environment can be resolved (UNDP 2019). By using decision support systems that produces educational system in line with the students' profiles the objective of each of these learnings can be achieved.

\subsection{Academic Adaptation}

Learning to live together

Based on historical information and students' knowledge and intelligent algorithms, this system can provide an adequate environment to the student to work their projects together 
and interact positively. The workgroups can be generated based on cultural complementarities. Also, system can provide professors with conflict resolution practices based on the students' background or culture.

\section{Learning to know}

The decision support system can provide students with many success stories that have been implemented in the sector of the activity related to the student specialization. Those stories can have a dual task. First by motivating the students to work during their academic program to validate the condition to obtain the degree. Second by forcing the students to think out of the box and innovate while conducting their projects and acquiring experiences.

\section{Learning to do}

Learning enough or learning to do enough is not enough. Students should be able to apply the acquired knowledge in complex situations. By using the decision support systems and by analyzing the available information, students can be provided simulators that produce real situation and real-life examples.

\section{Learning to be}

The system also should be students centric to detect their soft skills and provide them with an adapted training program to enhance what is needed.

Learning what and how to learn should be added to these four (Mercier-Laurent, 2011).

\subsection{Logistic Services}

In aim preserving balance of educational ecosystem, in addition to the academic support and adaptation, the welcome services should be provided to overcome the problems that might affect the academic track of students. Also, there should be a strong connection with the real life. This logistics support should include pre-arrival, on arrival and post arrival services to facilitate the integration of students in the desired country and reduce homesickness. The pre-arrival services should include finding accommodation, visa procedures guidance, organizing the airport pickups. On arrival is to make sure students are well settled down and have already all the administrative procedures related to the desired country.

Our decision support system includes the base of problem solving that have been addressed with previous batches/countries to learn from the previous experience. A mentorship system based on cultural affinity could be produced to assure the different groups of students coming from different cultures (Bakhtiari, 2011). Also, events should be organized to help the international student meet national students to exchange experiences at the 
language level, academic level, political level and cultural level as well. Such type of communication can diminish international student strains and help national student accept and understand the behavior of international students.

By applying those best practices, the number of international students in any country will grow and the possibility of having more comfortable international environment will be higher. This will reduce directly the risks and drawbacks that might be produced to globalization in education.

\section{Elements of Success of International Programs}

\section{Ecosystem of Students, Environment \& Staff}

The designed academic programs that are proposed to international students should be attractive and motivating not only for the students but also for the faculty members who are teaching the programs (Zhou 2018). When a student is seeking a degree in a certain country, the aim is to peruse a degree with an international staff and in an international environment and not only national. This is richer and more beneficial to international students.

The admission system described in section 2 can be used to recruit faculty members as well. The main objective is to have international staff able to adapt the teaching method to the students needs. The faculty members should focus on the success of international students in the desired country and program. By using this approach, we can solve of one the important problems, retaining the maximum number of international faculty and limit the turnover of good and adequate profiles.

This will have also a positive impact on national students as it helps them to be more open to accept cultural differences and to be more efficient in tackling professional life challenges and managing projects in a global environment. There will be an indirect leverage of national students' skills.

\section{Market absorption}

Many countries do not manage their intellectual assets, neither they do not have a talents management system. A very dangerous burden of globalization in education is when the economy of the desired country is not able to absorb the potential graduates. The objective is to shorten the distance between the fresh graduates and the recruiters and provide the adequate profiles to the market. This is a result of using an adapted approach with international students. It starts by providing an adapted curriculum lectured by international faculty members and working closely with companies. Also, offering local language 
courses throughout the whole academic program will help the students' integration easily the professional environment and valorize their degree obtained.

\section{Conclusion and Future Work}

Decision Support Systems described above helps recruiting motivated students and reducing risks incurred by globalization. It covers three main areas: admission of international students, the balance of educational ecosystem and success of educational program. Apparently, the provided analysis and proposed systems can help in reducing failure in the pursued programs and can reduce the unemployment of students seeking careers abroad, all of that in an acceptable and comfortable environment.

The Future work includes integration of components and testing on hundreds of students' applications to verify their outputs and validate the proposed algorithm. In fine we would like to connect this system with the Intellectual Capital Management in aim of planning the resource for the future, especially in IT field moving very quickly.

\section{References}

FT (2019) http://lexicon.ft.com/Term?term=globalisation

Ordonez, Edvinsson (2015) Intellectual Capital in Organizations, Eds Ordonez de Pablos P. and Edvinsson L., Routledge, 2015

Nguyen, Haddawy ( 2007) Decision Support System for Evaluating International Student, Nguyen T., Haddawy P. A, Frontier in Education Conference, DOI 10.1109/FIE.2007.4417958

Bakhtiari (2011) Globalization and Education: Challenges and Opportunities, Bakhtiari Sadegh, February 2011

Min Edu (2019) http://www.enseignementsup-recherche.gouv.fr/cid20200/etudiantsetrangers-inscriptions-dans-l-enseignement-superieur-francais.html

Szymankiewicz (2005) Conditions d'inscription et d'accueil des étudiants étrangers dans les universités, Rapport MESR 2005-023

UNDP (2019) https://www.undp.org/content/undp/en/home/sustainable-developmentgoals/goal-4-quality-education.html

Mercier-Laurent (2011) Innovation Ecosystems, Wiley

Zhou (2018) Retaining International Faculty, Jiangyuan Zhou, December 2018 